\title{
AMENABLE ACTIONS OF GROUPS
}

\author{
SCOT ADAMS, GEORGE A. ELLIOTT, AND THIERRY GIORDANO
}

\begin{abstract}
The equivalence between different characterizations of amenable actions of a locally compact group is proved. In particular, this answers a question raised by R. J. Zimmer in 1977.
\end{abstract}

\section{INTRODUCTION}

The concept of amenability for a second countable locally compact group $G$ can be described in many different equivalent ways. Two of the most wellknown characterizations are the following:

(i) there is a left invariant mean on $L^{\infty}(G)$;

(ii) any affine $G$-action has a fixed point.

In [Z1], R. J. Zimmer introduced the notion of amenability for an action of a separable locally compact group $G$, or for an equivalence relation, on a standard Borel space $(S, \mu)$ with a probability measure. He used an analogue of the fixed point criterion.

In [CW], A. Connes and E. J. Woods studied group-invariant, time-dependent Markov random walks. In particular, if $(X, \nu)$ denotes the Poisson boundary of a group-invariant matrix-valued random walk on a separable locally compact group, they pointed out that there exists a $G$-equivariant conditional expectation from $L^{\infty}(X \times G)$ onto $L^{\infty}(X)$.

If $G$ is discrete, it is known among other equivalent characterizations that the action of $G$ on $(S, \mu)$ is amenable if and only if there exists a $G$-equivariant conditional expectation from $L^{\infty}(S \times G)$ onto $L^{\infty}(S)$, and if and only if $(S, \mu)$ is isomorphic as a $G$-space to the Poisson boundary of a group-invariant matrixvalued Markov random walk on $G$.

In this paper we shall extend these equivalences to second countable locally compact groups in general. We summarize these different equivalences in the following theorem:

Theorem A. If $(S, \mu)$ is a standard measure space and $G$ acts ergodically on $(S, \mu)$, then the following statements are equivalent:

(i) $(S, \mu)$ is an amenable $G$-space;

Received by the editors September 13, 1993.

1991 Mathematics Subject Classification. Primary 47A35, 28D99.

Research of the first author supported by NSF Postdoctoral Grant No. DMS-9007248.

Research of the second author supported in part by operating grants from NSERC. 
(ii) $(S, \mu)$ is isomorphic as a $G$-space to the Mackey range of a homomorphism from a countable amenable equivalence relation to $G$;

(iii) $(S, \mu)$ is isomorphic as a $G$-space to the Poisson boundary of a groupinvariant matrix-valued Markov random walk on $G$;

(iv) there exists a G-equivariant conditional expectation from $L^{\infty}(S \times G)$ onto $L^{\infty}(S)$.

(v) the equivalence relation $\mathscr{R}_{G}$ induced by the action of $G$ on $(S, \mu)$ is amenable and the stability subgroup $G_{s}=\{g \in G ; s g=s\}$ is amenable $\mu$-a.e.

In [Z2], Zimmer proves that (ii) implies (i). The converse of this implication was shown by Golodets and Sinel'shchikov in [GS]. In their announcement, Golodets and Sinel'shchikov state and give an outline of the proof of the following implications:

$$
\text { (i) } \Rightarrow \text { (ii) } \Rightarrow \text { (v) } \Rightarrow \text { (i) . }
$$

In [Z3], Zimmer proved that if $G$ is a discrete countable group then (iv) implies (i). His proof works without any change in the continuous case.

We sketch the proof of Golodets and Sinel'shchikov that (i) implies (ii) in $\S 1$, and use it as a starting point in $\S 2$ to prove the implication from (ii) to (iii). Using results of Jaworski's thesis [J], we then deduce (iv) from (iii).

In $\S 3$, we give a direct proof of (i) to (iv), which we use in $\S 4$ to show that if (i) holds then the stability subgroups $G_{s}$ are amenable a.e. As the amenability of $\mathscr{R}_{G}$ follows from the definitions, the implication (i) to $(v)$ is proved. In $\S 5$, we show the converse, i.e., (v) implies (i). Note that Golodets and Sinel'shchikov gave in [GS] another proof of this equivalence using (ii).

Using the main result of [CFW], we can show that a countable equivalence relation is amenable if and only if almost every ergodic component is amenable. Techniques of [FHM] extend this to any equivalence relation generated by an action of a locally compact group. Thus assertion ( $v$ ) has the following consequence:

Corollary B. Let $G$ be a locally compact, second countable topological group acting nonsingularly on a standard measure space $(X, \mu)$. Let $\Phi: X \rightarrow E$ be a map defining a decomposition of $X$ into ergodic components. Let $\nu=\Phi_{*}(\mu)$ and let $\left\{\mu_{e}\right\}_{e \in E}$ be a disintegration $\mu$ along fibers of $\Phi$; then, for $\nu$-a.e. $e \in E$, the $G$-action on $\left(X, \mu_{e}\right)$ is ergodic. The action of $G$ on $X$ is amenable if and only if, for $\nu$-a.e. $e \in E$, the action of $G$ on $\left(X, \mu_{e}\right)$ is amenable.

This result is not surprising, but does not seem to follow directly from the definition of amenability. The same may be said of the following assertion.

Corollary C. Let $G$ be a locally compact, second countable topological group acting nonsingularly on the standard measure spaces $(X, \mu)$ and $(Y, \nu)$. Let $\Phi: X \rightarrow Y$ be a $G$-equivariant map and assume that $\Phi_{*}(\mu)=\nu$. If the $G$-action on $Y$ is amenable, then the $G$-action on $X$ is amenable.

In other words, an extension of an amenable action is again amenable. This is the dynamical analogue of the statement that a closed subgroup of an amenable group is again amenable.

Corollary $\mathrm{C}$ extends [Z1], Theorem 2.4 to actions which are not necessarily ergodic. The proof is straightforward, given Corollary B and [ $\mathrm{Z1}$ ], Theorem 2.4, and given the fact that almost every ergodic component of $X$ is an extension of an amenable ergodic component of $Y$. 
In Lemma 3.15, we borrow an idea from $\mathrm{R}$. Lyons and in Lemma 4.3 one from J. King. The first author would like to thank J. Feldman and T. Steger and the third one W. Jaworski for helpful conversations.

\section{The CONSTRUCtion OF Golodets AND Sinel'SHCHikov}

In [GS], Golodets and Sinel'shchikov announced and gave a sketch of the proof of the following result:

Theorem 1.1. Let $(S, \mu)$ be a standard measure space and let $G$ be a second countable locally compact group acting ergodically and amenably on $(S, \mu)$.

Then $(S, \mu)$ is isomorphic as a $G$-space to the Mackey range of a homomorphism from a countable amenable equivalence relation to $G$.

For the convenience of the reader, we present an outline of the proof.

An important tool in the proof of 1.1 is the following fact, used without comment by Golodets and Sinel'shchikov. We prove it for the sake of completeness. A different and more complicated proof is given in [AS], Proposition 4.4.

Proposition 1.2. For any second countable locally compact group $G$, there exists a standard measure $G$-space $(X, \nu)$ such that

(a) $\nu$ is a G-invariant probability measure,

(b) the action of $G$ is essentially free.

Let us first state the following technical result:

Lemma 1.3. Let $\Omega$ be a standard Borel $G$-space, with a $G$-invariant probability measure $\mu$. Then there exist a compact metric space $Y$, on which $G$ acts continuously, and a G-invariant probability measure $\nu$, whose support is $Y$, such that $L^{2}(\Omega, \nu)$ and $L^{2}(Y, \nu)$ are $G$-isomorphic.

Proof. By [V], Theorem 5.7, there is a compact metric space $X$ on which $G$ acts continuously and an injective $G$-map $\psi: \Omega \rightarrow X$. The measure $\nu=$ $\psi(\mu)$ is a $G$-invariant probability measure on $X$ and its support is a closed $G$-invariant subset of $X$. Set

$$
(Y, \nu)=(\operatorname{Supp}(\nu), \nu \mid \operatorname{Supp}(\nu)) \text {. }
$$

As $L^{2}(\Omega, \nu)=L^{2}(Y, \nu)$, the lemma is proved.

To prove 1.2, we need the following construction. Let $H$ be a (separable) real Hilbert space and let $\pi$ denote an orthogonal representation of $G$ on $H$. As in Section 5.2.13 of [Z4], we consider the probability space

$$
(\Omega, \nu)=\prod_{n \geq 1}\left(\mathbb{R}, \frac{1}{\sqrt{2 \pi}} \exp \left(-\frac{x^{2}}{2}\right) d x\right) .
$$

Let $T: H \rightarrow L^{2}(\Omega, \nu)$ denote the orthogonal isometry from $H$ into $L^{2}(\Omega, \nu)$ defined by $T e_{n}=p_{n}$ for $n \geq 1$, where $\left(e_{n}\right)_{n \geq 1}$ is a basis for $H$ and $p_{n}$ is the projection onto the $n$th component of $\Omega$. Then there exists an action $\eta$ of $G$ on $\Omega$ preserving the measure $\mu$ such that the following diagram commutes:

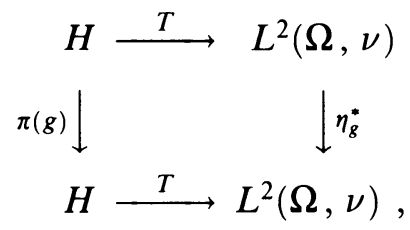


where for $f \in L^{2}(\Omega, \nu)$,

$$
\left(\eta_{g}^{*} f\right)(\omega)=f\left(\eta\left(g^{-1}\right) \omega\right), \quad \omega \in \Omega .
$$

Moreover, we may assume that $(\Omega, \mu)$ and $\eta$ have the properties of Lemma 1.3. Then, keeping the above notations, we have:

Lemma 1.4. If $\pi$ is a faithful representation, then for all $g \in G, g \neq 1$, there exist a neighourhood $N$ of $g$ and a nonempty open set $U$ of $\Omega$ such that

$$
\eta_{h}(U) \cap U=\varnothing, \quad h \in N .
$$

Proof. Let $g \in G, g \neq 1$, be given. As $\pi$ is faithful, there exists $\xi \in$ $H,\|\xi\|=1$, such that $\pi\left(g^{-1}\right) \xi \neq \xi$. Set $f=T(\xi) \in L^{2}(\Omega, \nu)$ and $A_{g}=\left\{\omega \in \Omega ; \eta_{g} \omega \neq \omega\right\}$. As $\pi\left(g^{-1}\right) \xi \neq \xi, A_{g}$ is a nonempty open set. Fix $\omega \in A_{g}$, and let $V$ be a open neighbourhood of $\omega$ such that $\eta_{g}(V) \cap V=\varnothing$. By continuity of $\eta$, there exists a open neighbourhood $W$ of $\omega$ such that

$$
\eta_{h}(W) \subset \eta_{g}(V), \quad h \in N_{g} \text {. }
$$

Then $W \cap V$ is an open neighbourhood of $\Omega$ such that with $W \cap V=U$,

$$
\eta_{h}(U) \cap U \subset \eta_{g}(V) \cap V=\varnothing, \quad h \in N_{g} .
$$

Proof of Proposition 1.2. Keeping the above notation, we denote by $(X, \nu)$ the infinite product space $\prod_{n \geq 1}(\Omega, \mu)$ and consider on $(X, \nu)$ the $G$-action given by:

$$
g x=g\left(\omega_{1}, \cdots, \omega_{n}, \cdots\right)=\left(\eta_{g} \omega_{1}, \cdots, \eta_{g} \omega_{n}, \cdots\right)
$$

for $g \in G$ and $x=\left(\omega_{1}, \cdots, \omega_{n}, \cdots\right) \in X$.

Then this action of $G$ preserves the measure $\nu$. By Lemma 1.4, there exists a neighbourhood $N_{g}$ and a nonempty open set $U_{g}$ such that

$$
\begin{aligned}
\{x \in & \left.X ; \exists h \in N_{g}, h x=x\right\} \\
& =\left\{x=\left(\omega_{n}\right)_{n \geq 1} \in X ; \exists h \in N_{g}, \eta_{g} \omega_{n}=\omega_{n}, \forall n \geq 1\right\} \\
& \subseteq \prod_{n \geq 1} \Omega \backslash\left\{\omega \in \Omega ; \eta_{h} \omega \neq \omega, \forall h \in N_{g}\right\} \\
& \subseteq \prod_{n \geq 1} \Omega \backslash U_{g} .
\end{aligned}
$$

As $G$ is second countable locally compact, there exists $\left(g_{n}\right)_{n \geq 1}$ such that

$$
\bigcup_{n \geq 1} N_{g_{n}}=G \backslash\{1\} .
$$

If $G_{x}=\{g \in G ; g x=x\}$ denotes the stabilizer of $x \in X$, then

$$
\begin{aligned}
\left\{x \in X ; G_{x} \neq\{1\}\right\} & =\{x \in X ; \exists g, g \neq e, g x=x\} \\
& =\bigcup_{k \geq 1}\left\{x \in X ; \exists h \in N_{g_{k}}, h x=x\right\} \\
& \subseteq \bigcup_{k \geq 1}\left(\prod_{n \geq 1} \Omega \backslash U_{g_{k}}\right) .
\end{aligned}
$$

As $\nu\left(\bigcup_{k \geq 1}\left(\prod_{n \geq 1} \Omega \backslash U_{g_{k}}\right)\right) \leq \sum_{k \geq 1} \prod_{n \geq 1} \mu\left(\Omega \backslash U_{g_{k}}\right)=0$, the action of $G$ on $X$ is essentially free. 
Proposition 1.5. Let $(S, \mu)$ be a standard measure G-space.

Then $(S, \mu)$ is isomorphic as a G-space to the Mackey range of a homomorphism from an equivalence relation to $G$.

Proof. Let $(X, \nu)$ be a free $G$-space with an invariant probability measure, as given by 1.2 .

On $(Y, \lambda)=\prod_{n \in \mathbb{Z}}(X, \nu)$, we consider the following actions of $G$ and $\mathbb{Z}$ :

$$
\begin{aligned}
& y g=\left(x_{1}, \cdots, x_{n}, \cdots\right) g=\left(x_{1} g, \cdots, x_{n} g, \cdots\right), \quad g \in G, \\
& n y=S^{n} y, \quad n \in \mathbb{Z},
\end{aligned}
$$

where $S$ denotes the shift on $(Y, \lambda)$.

These two actions commute and therefore $(Y, \lambda)$ is a $(G \times \mathbb{Z})$-space. Then $(S \times Y, \mu \times \lambda)$ becomes a $(G \times \mathbb{Z})$-space, via

$$
(s, y)(g, n)=(s g, n y g) \text { for }(s, y) \in S \times Y \text { and }(g, n) \in G \times \mathbb{Z} .
$$

Consider the cocycle defined by

$$
\begin{aligned}
\alpha:(S \times Y, \mu \times \lambda) \times(G \times \mathbb{Z}) & \rightarrow G \\
((s, y),(g, n)) & \mapsto g .
\end{aligned}
$$

Let us still denote by $\alpha$ the $(G \times \mathbb{Z})$-skew-product action on $S \times Y \times G$, i.e., for $(g, n) \in G \times \mathbb{Z}$,

$$
(s, y, h)(\alpha(g, n))=(s g, n y g, h g), \quad(s, y, h) \in S \times Y \times G .
$$

If $p: S \times Y \times G \rightarrow S$ is defined by $p(s, y, g)=s g^{-1}$, then it induces a $G$-isomorphism between $L^{\infty}(S, \mu)$ and $L^{\infty}(S \times Y \times G)^{\alpha}$, because $G$ acts freely on $S \times Y$ and $\mathbb{Z}$ acts ergodically on $Y$.

Proof of Theorem 1.1. Let us keep the notation introduced in the proof of 1.5. As $(S, \mu)$ is an amenable $G$-space, the space $(S \times Y, \mu \times \lambda)$ is $G$-amenable by [Z1], Proposition 4.3 .4 , and hence $(G \times \mathbb{Z})$-amenable by [GS1], Theorem A.1.

By [CFW], Corollary 16, the ergodic equivalence relation obtained from the orbits of $G \times \mathbb{Z}$ is hyperfinite. Then using [FHM], Theorem 6.4, we get the result.

Remark 1.6. (i) In [Z1], Theorem 3.3, Zimmer proves the converse of Theorem 1.1 .

(ii) Let $(S, \mu)$ be a standard measure space and let $G$ be a second countable locally compact group acting ergodically on $(S, \mu)$. Let $\mathscr{R}_{G}$ denote the equivalence relation on $(S, \mu)$ induced by $G$. Using Theorem 1.1, Golodets and Sinel'shchikov prove in [GS] that $(S, \mu)$ is an amenable $G$-space if and only if $\mathscr{R}_{G}$ is amenable and the stabilizers $G_{s}$ are amenable, $\mu$-a.e. In $\S \S 4$ and 5 , we shall give a direct proof of this result.

\section{Mackey Range AND PoIsSON Boundary}

In this section, $G$ will denote as above a second countable locally compact group. We refer to [CW] or [EG] for the notion of Poisson boundary used here. The main result of this section is the following: 
Theorem 2.1. Let $\mathscr{R}$ be an ergodic amenable discrete equivalence relation on a standard Borel space $(Y, \lambda)$ and let $\alpha: \mathscr{R} \rightarrow G$ be a homomorphism.

Then the Mackey range of $\alpha$ is isomorphic (as a G-space) to the Poisson boundary of a matrix-valued random walk on $G$.

This result extends Theorem 3.1 of [EG] and its proof to continuous groups. For the convenience of the reader let us recall the following definition ([EG], Definition 3.4, or [S3]).

Definition 2.2. (1) A Bratteli diagram $D$ is a graph with set of vertices $V$ and set of edges $E$ with the following properties :

(a) $V$ is the disjoint union of subsets $V(n)(n \geq 0)$ with $|V(n)|<\infty$ for all $n \geq 0$.

(b) $E$ is the disjoint union of subsets $E(n)(n \geq 0)$, with each edge $e \in E(n)$ connecting a vertex $s(e) \in V(n)$ with a vertex $r(e) \in V(n+1)$.

(c) For every vertex $v \in V$, there exist $e, f \in E$ with $s(e)=v, r(f)=v$ (except for $v \in V(0)$, for which we omit the second requirement).

(2) A path in $D$ is a sequence $\left(e_{k}\right)$ of edges with $s\left(e_{0}\right) \in V(0)$, and $s\left(e_{k}\right)=$ $r\left(e_{k-1}\right), k \geq 1$.

We denote by $\Omega_{n}$ the space of paths of length $n$, and by $\Omega$ the space of paths of infinite length. We view $\Omega$ as a topological space, with basis $\{\Omega(f) ; f \in$ $\left.\Omega_{n}, n \geq 1\right\}$, where to each $f=\left(f_{0}, f_{1}, \cdots, f_{n-1}\right) \in \Omega_{n}$ we associate the set

$$
\Omega(f)=\left\{e \in \Omega, e_{k}=f_{k} ; 0 \leq k \leq n\right\} .
$$

(3) An AF-measure (or Markov measure) $\mu_{p}$ on $\Omega$ is a measure determined by a system of transition probabilities $p$ (i.e. maps $p: E \rightarrow[0,1]$ with $p(e) \geq$ 0 and $\sum_{\{e, s(e)=v\}} p(e)=1$ for every vertex $\left.v\right)$, given by

$$
\mu_{p}(\Omega(f))=\prod_{k=0}^{n} p\left(f_{k}\right),
$$

where $f=\left(f_{0}, f_{1}, \cdots, f_{n}\right) \in \Omega_{n}$.

Note that $\Omega$ carries a canonical equivalence relation $\mathscr{R}_{\Omega}$ defined by

$$
e \mathscr{R}_{\Omega} f \Leftrightarrow \text { for some } n, e_{k}=f_{k} \text { for all } k \geq n \text {. }
$$

If $\left(b_{n}\right)_{n \geq 0}$ is a sequence of maps $b_{n}: E(n) \rightarrow G$, let us denote by $\beta^{\prime}$ : $\mathscr{R}_{\Omega} \rightarrow G$ the homomorphism given by

$$
\boldsymbol{\beta}^{\prime}(\boldsymbol{e}, f)=b_{0}\left(e_{0}\right) \cdots b_{n}\left(e_{n}\right) b_{n}\left(f_{n}\right)^{-1} \cdots b_{0}\left(f_{0}\right)^{-1}
$$

whenever $e \mathscr{R}_{\Omega} f$ and $e_{k}=f_{k}$ for $k \geq n+1$.

Proof of Theorem 2.1. Let $X$ denote the compact group $\prod_{n>1}\{0,1\}$ with the Borel $\sigma$-algebra $\mathscr{A}$ and denote by $K$ the dense subgroup $\bigoplus_{n>1} \mathbb{Z} / 2 \mathbb{Z}$ acting on $X$ by addition. For all $k \geq 1$, let us denote by $S_{k}$ the automorphism of $X$ corresponding to the element $\left(x_{n}\right)_{n \geq 1} \in K$ with $x_{n}=\delta_{n, k}, \quad n \geq 1$.

By Theorem 10 of [CFW] and [C], Lemma 7, we may assume that the ergodic amenable dynamical system $(Y, \lambda, \mathscr{R})$ is equivalent to $\left(X, \mu, \mathscr{R}_{K}\right)$ where $\mu$ is a $K$-ergodic nonatomic probability measure on $(X, \mathscr{A})$ such that

$$
\text { for each } k \geq 1, \quad \log \frac{d \mu S_{k}}{d \mu} \quad \text { takes only finitely many values, }
$$


and $\mathscr{R}_{K}$ is the equivalence induced by the action of $K$.

By Lemma 3.5 of [EG], there exist

(i) a Bratteli diagram $D$, an AF-measure $\mu_{p}$ on the path space $\Omega$ of $D$, and an isomorphism $\psi:(X, \mu) \rightarrow\left(\Omega, \mu_{p}\right)$ such that $\psi(\mu)=\mu_{p}$ and

$$
\psi(x) \mathscr{R}_{\Omega} \psi(y) \Leftrightarrow x \mathscr{R}_{K} y,
$$

(ii) a sequence $\left(b_{n}\right)_{n \geq 0}$ of maps $b_{n}: E(n) \rightarrow G$ such that if $\beta^{\prime} \in Z^{1}\left(\mathscr{R}_{\omega}, G\right)$ denotes the cocycle given by

$$
\beta^{\prime}(e, f)=b_{0}\left(e_{0}\right) \cdots b_{n}\left(e_{n}\right) b_{n}\left(f_{n}\right)^{-1} \cdots b_{0}\left(f_{0}\right)^{-1}
$$

whenever $e \mathscr{R}_{\Omega} f$ and $e_{k}=f_{k}$ for $k \geq n+1$, then

$$
\beta\left(x, x S_{k}\right)=\beta^{\prime}\left(\psi(x), \psi\left(x S_{k}\right)\right) \quad \mu \text {-a.e. }, k \in \mathbb{N} \text {. }
$$

For each $n \geq 0$ and each $(v, w) \in V(n) \times V(n+1)$, consider the positive measure $\sigma_{n, v, w}$ on $G$ given by

$$
\sigma_{n, v, w}=\sum_{\{e \in E(n) ; s(e)=v, r(e)=w\}} p(e) \delta_{b_{n}(e)}
$$

where $\delta_{b_{n}(e)}$ is the Dirac function at $g=b_{n}(e)$. Note that, for every $v \in G$,

$$
\sum_{w \in V(n+1)} \sigma_{n, v, w}(G)=\sum_{w \in V(n+1)} \sum_{\{e \in E(n) ; s(e)=v, r(e)=w\}} p(e)=1 .
$$

For each $n \geq 0$, set $F_{n}=\bigsqcup_{v \in V(n)} G$ and consider the transition probability $P_{n+1}^{n}$ from $F_{n}$ to $F_{n+1}$ given by

$$
P_{n+1}^{n}((v, g),(w, A))=\sigma_{n, v, w}\left(g^{-1} A\right) .
$$

Let us fix a countable dense subgroup $\Gamma$ of $G$. By [GS2], Proposition 1.2, we may assume that $\alpha$ takes values in $\Gamma$, and by using [EG], Lemma 3.3, there exists a homomorphism $\beta: \mathscr{R}_{K} \rightarrow \Gamma \subset G$ cohomologous to $\alpha$, such that for every $\gamma \in K$, the map $x \mapsto \beta(x, x \gamma)$ takes only finitely many values.

Then the Markov process consisting of the sequence of measurable spaces $\left(F_{n}\right)_{n \geq 0}$ with the transition probabilities $\left(P_{n}^{n-1}\right)_{n \geq 1}$ defines a right groupinvariant matrix-valued random walk on $G$ associated with the sequence $\left(\sigma_{n}\right)_{n \geq 1}$. Therefore Theorem 2.1 is proved.

Corollary 2.3. Let $(S, \mu)$ be a standard measure space and $G$ be a second countable locally compact group acting ergodically and amenably on $(S, \mu)$.

Then there exists a conditional expectation

$$
P: L^{\infty}\left(S \times G, \mu_{G} \times \mu\right) \rightarrow L^{\infty}(S, \mu)
$$

such that for all $h \in G$,

$$
P(f \cdot h)=P(f) h, \quad f \in L^{\infty}\left(S \times G, \mu_{G} \times \mu\right),
$$

where $f \cdot h(s, g)=f .(s h, g h),(s, g) \in S \times G$.

Proof. By Theorem 2.1, we may assume that $(S, \mu)$ is the Poisson boundary of a matrix-valued random walk on $G$.

Therefore, 2.3 follows from [J], Theorem 2.3.4. 


\section{EQUIVARIANT CONDITIONAL EXPECTATIONS}

A direct proof of the implication from (i) to (iv) of Theorem A will be presented in this section.

To begin we prove some technical results that we will need later.

Let $M$ be a von Neumann algebra, and denote by $M_{*}$ the predual of $M$ and by Aut $(M)$ the group of automorphisms of $M$.

Let $U: G \rightarrow \operatorname{Aut}(M)$ be a representation of $G$ into $M$ (i.e. for all $x \in M$ and all $\phi \in M_{*}$, the map $g \mapsto \phi\left(U_{g} x\right)$ is continuous).

For all $\mu \in M(G)$, the integral

$$
\int_{G} U_{g}(x) d \mu(g)
$$

defines a bounded linear form on $M_{*}$, hence an element of $M$, denoted by $U(\mu)(x)$.

Let $B(M)$ denote the Banach algebra of all bounded linear operators on $M$. For $x \in M$ and $\phi \in M_{*}$, we define a bounded linear form $\phi(\cdot x)$ on $B(M)$ by

$$
\phi(\cdot x)(T)=\phi(T x), \quad T \in B(M) .
$$

Denote by $B(M)_{*}$ the norm-closed linear subspace

$$
\overline{\operatorname{Span}\left\{\phi(\cdot x) ; \phi \in M_{*}, x \in M\right\}} \subset B(M)^{*} \text {. }
$$

Remark 3.1. For $S \in B(M)$, the maps $T \in B(M) \mapsto S T \in B(M)$ and $T \in$ $B(M) \mapsto T S \in B(M)$ are $\sigma\left(B(M), B(M)_{*}\right)$-continuous.

The following result is easy to check:

Lemma 3.2. If $\left(\mu_{n}\right)_{n \geq 1}$ and $\mu$ are measures with compact support and if $\mu_{n} \rightarrow \mu$ weakly, then $U\left(\mu_{n}\right)$ converges $\sigma\left(B(M), B(M)_{*}\right)$ to $U(\mu)$.

Let us denote by $B(M)_{1}$ the closed unit ball of $B(M)$. By the BanachAlaoglu theorem, $B(M)_{1}$ is $\sigma\left(B(M), B(M)_{*}\right)$-compact. Therefore we have:

Lemma 3.3. Let $\mathscr{R} \subset B(M)_{1}$. Then for all $x \in M$,

$$
\overline{\mathscr{R}}^{\sigma\left(M, M_{*}\right)}=\left(\overline{\mathscr{R}}^{\sigma\left(B(M), B(M)_{*}\right)}\right) x .
$$

Proof. It follows from the definitions that $\overline{\mathscr{R}} x \subset \overline{\mathscr{R} x}$. Let $y \in \overline{\mathscr{R} x}$ and let $\left\{\Phi_{\alpha}\right\}$ be a net in $\mathscr{R}$ such that $\Phi_{\alpha} x \rightarrow y$. By the Banach-Alaoglu theorem, $\overline{\mathscr{R}}$ is compact, and so after passing to a subnet, we may assume that $\left\{\Phi_{\alpha}\right\}$ converges, say to $\Phi \in \overline{\mathscr{R}}$. Then $\Phi_{\alpha} x \rightarrow \Phi x$, and so $y=\Phi x \in \overline{\mathscr{R}} x$.

From now on, $(G, \rho)$ will be a locally compact, second countable topological group, with a right invariant Haar measure $\rho$. Let us state the main theorem of this section.

Theorem 3.4. Let $(S, \mu)$ be a standard measure space and $G$ be a locally compact, second countable topological group, acting amenably on $(S, \mu)$.

Then there exists a conditional expectation $P: L^{\infty}(S \times G, \mu \times \rho) \rightarrow L^{\infty}(S, \mu)$ such that $P(f \cdot g)=(P f) \cdot g$, where

$$
f \cdot g(s, h)=f(s g, h g) \text { and }(P f) \cdot g(s)=P f(s g), \quad s \in S, g \in G \text {. }
$$


Let us denote by $R$ the representation of $G$ on either $L^{\infty}(S \times G)$ or $L^{\infty}(S, \mu)$ given for $g \in G$ by

$$
R_{g} f(s, h)=f(s g, h g) \quad \text { if } \quad f \in L^{\infty}(S \times G)
$$

and

$$
R_{g} f(h)=f(h g) \quad \text { if } \quad f \in L^{\infty}(G) .
$$

For $g \in G$, let also $V_{g}$ be defined by

$$
V_{g} f(s, h)=f\left(s, g^{-1} h\right) \quad \text { if } f \in L^{\infty}(S \times G),
$$

and

$$
V_{g} f(h)=f\left(g^{-1} h\right) \quad \text { if } \quad f \in L^{\infty}(G) .
$$

As $L^{\infty}(S \times G) \cong L^{\infty}(S) \otimes L^{\infty}(G)$, it is easy to check that the fixed point subalgebra

$$
L^{\infty}(S \times G)^{V}=\left\{f \in L^{\infty}(S \times G) ; V_{g} f=f \text { for all } g \in G\right\}
$$

is canonically isomorphic to $L^{\infty}(S)$.

Lemma 3.5. The map $I: S \times G \rightarrow S \times G$ defined by $I(s, h)=\left(s h^{-1}, h^{-1}\right)$ intertwines the actions $R$ and $V$, i.e., for all $g \in G$, for all $s \in S$,

$$
I \circ R_{g}(s, h)=V_{g} \circ I(s, h) \text { and } I \circ V_{g}(s, h)=R_{g} \circ I(s, h) \text {. }
$$

Using Lemma 3.5, one sees that Theorem 3.4 is equivalent to the following:

Theorem 3.6. Let $(S, \mu)$ be a standard measure space and $G$ be a locally compact, second countable topological group, acting amenably on $(S, \mu)$. Then there exists a conditional expectation $\Phi$ of $L^{\infty}(S \times G)$ onto $L^{\infty}(S \times G)^{R}$ which is $V_{g}$-equivariant for all $g \in G$.

The proof of this theorem will be divided into a series of lemmas.

Let us denote by $B(S \times G)$ the set of bounded Borel functions on $S \times G$.

Definition 3.7. A function $f \in B(S \times G)$ is uniformly continuous on R-orbits if for any $\epsilon>0$, there exists a neighbourhood $U$ of the identity in $G$ such that, for all $g \in U$,

$$
|f(s g, h g)-f(s, h)|<\epsilon \text { for all }(s, h) \in S \times G .
$$

Remark 3.8. Let $f \in B(S \times G)$ be uniformly continuous on $R$-orbits. Then, for all $g \in G, R_{g} f$ is again uniformly continuous on $R$-orbits.

Let us denote by $C_{c}(G)$ the continuous functions on $G$ with compact support.

Lemma 3.9. If $\phi \in C_{c}(G)$, then any function class in

$$
R_{\phi}\left(L^{\infty}(S \times G)\right)=\left\{\int R_{g} f(\cdot, \cdot) \phi(g) d \rho(g) ; f \in L^{\infty}(S \times G)\right\}
$$

contains a function in $B(S \times G)$ which is uniformly continuous on R-orbits.

Proof. Let $f \in L^{\infty}(S \times G)$ be given. Choose $f_{0} \in B(S \times G)$ such that

$$
f_{0}=f \text {, a.e. }
$$


Set $M=\sup \left\{\left|f_{0}(s, h)\right| ;(s, h) \in S \times G\right\}$, and let $U$ be a neighbourhood of the identity in $G$ such that

$$
\int_{G}\left|\phi\left(k g^{-1}\right)-\phi(k)\right| d \rho(k)<\frac{\varepsilon}{M} \quad \text { for all } g \in U \text {. }
$$

For $g \in G$, we have

$$
\begin{aligned}
R_{g} R_{\phi}\left(f_{0}\right)(s, h) & =\int R_{g} f_{0}(s k, h k) \phi(k) d \rho(k) \\
& =\int f_{0}(s k, h k) \phi\left(k g^{-1}\right) d \rho(k), \quad(s, h) \in S \times G .
\end{aligned}
$$

Then, for all $g \in U$,

$$
\begin{aligned}
\left|R_{g} R_{\phi}\left(f_{0}\right)(s, h)-R_{\phi}\left(f_{0}\right)(s, h)\right| & =\int\left|\left(f_{0}\right)(s k, h k)\right|\left|\phi\left(k g^{-1}\right)-\phi(k)\right| d \rho(k) \\
& <M \int_{G}\left|\phi\left(k g^{-1}\right)-\phi(k)\right| d \rho(k)<\varepsilon . \quad
\end{aligned}
$$

If $X$ is a normed space and $A \subset X$, we denote by co $(A)$ (resp. $\overline{c o}(A))$ the convex hull (resp. the closed convex hull) of $A$ in $X$.

Lemma 3.10. Let $f_{0} \in B(S \times G)$ be uniformly continuous on $R$-orbits and let $\Gamma$ be a dense subset of $G$. For $s \in S$, consider the subsets

$$
\left\{R_{\gamma} f_{0}(s, \cdot) ; \gamma \in \Gamma\right\} \subset\left\{R_{g} f_{0}(s, .) ; g \in G\right\} \subset L^{\infty}(G) .
$$

Then

$$
\overline{\mathrm{co}}\left\{R_{\gamma} f_{0}(s, \cdot) ; \gamma \in \Gamma\right\}=\overline{\mathrm{co}}\left\{R_{g} f_{0}(s, .) ; g \in G\right\} .
$$

Proof. As $\Gamma \subset G$, we have $\overline{\operatorname{co}}\left\{R_{\gamma} f_{0}(s, \cdot) ; \gamma \in \Gamma\right\} \subset \overline{\operatorname{co}}\left\{R_{g} f_{0}(s, \cdot) ; g \in G\right\}$. Conversely, fix $g \in G$ and choose a sequence $\left(\gamma_{n}\right)_{n \geq 1}$ in $\Gamma$ such that $\gamma_{n} \rightarrow g$.

Then,

$$
R_{\gamma_{n}} f_{0} \rightarrow R_{g} f_{0} \text { uniformly on } S \times G
$$

and so

$$
R_{\gamma_{n}} f_{0}(s, \cdot) \rightarrow R_{g} f_{0}(s, \cdot) \text { uniformly on } G
$$

Then,

$$
R_{g} f_{0}(s, \cdot) \in \overline{\left\{R_{\gamma} f_{0}(s, \cdot) ; \gamma \in \Gamma\right\}} \text {. }
$$

Let us denote by $L^{\infty}(S)_{+}$the set of nonnegative functions in $L^{\infty}(S)$.

Definition 3.11. Let $\mathscr{R} \subset B\left(L^{\infty}(S \times G)\right)$ denote the set of operators defined by $\left(\sum_{i=1}^{n} a_{i} R_{g_{i}} f\right)(s, h)=\sum_{i=1}^{n} a_{i}(s) f\left(s g_{i}, h g_{i}\right), \quad(s, h) \in S \times G, f \in L^{\infty}(S \times G)$, where for $1 \leq i \leq n$,
(1) $g_{i} \in G$,
(2) $a_{i} \in L^{\infty}(S)_{+}, \quad$ and
(3) $\sum_{i=1}^{n} a_{i}=1$

Remark 3.12. (a) As $L^{\infty}(S) \cong L^{\infty}(S \times G)^{V}$, (2) can be replaced by

(2') $a_{i} \in L^{\infty}(S \times G)_{+}^{V}$. 
(b) By definition, $\mathscr{R} \subset B\left(L^{\infty}(S \times G)\right)_{1}$ and by Lemma 3.2,

$$
\overline{\mathscr{R}} f=\overline{\mathscr{R} f} \text { for all } f \in L^{\infty}(S \times G) \text {. }
$$

Let $E$ denote the separable Banach space $L^{1}(G, \rho)$ and $c: S \times G \rightarrow \operatorname{Iso}(E)$ the cocycle given by

$$
c(s, g) f(h)=f(h g), \quad f \in L^{1}(G, \rho), \quad h \in G .
$$

If $H\left(E_{1}^{*}\right)$ denotes the group of homeomorphisms of the closed unit ball $E_{1}^{*}$ with the topology of uniform convergence, then the induced (Borel) cocycle $c^{*}: S \times G \rightarrow H\left(E_{1}^{*}\right)$ is defined by

$$
c^{*}(s, g) f(h)=\left(c(s, g)^{-1}\right)^{*} f(h)=f(h g), \quad f \in L^{\infty}(G), h \in G .
$$

Lemma 3.13. Let $f_{0} \in B(S \times G)$ be uniformly continuous on $R$-orbits and be such that $\left\|f_{0}\right\|_{L^{\infty}(S \times G)} \leq 1$. For $s \in S$, set $A_{s}=\overline{\mathrm{co}}\left\{R_{g} f_{0} ; g \in G\right\} \subset L^{\infty}(S \times G)$. Then

(i) $\left\{A_{s}\right\}_{s \in S}$ is a c-invariant Borel field of compact convex subsets of $L^{\infty}(G)_{1}$.

(ii) If $\lambda$ is a Borel section of this field, then there exists $f \in \overline{\mathscr{R} f_{0}}$ such that

$$
\lambda(s)=f(s, \cdot), \quad \text { a.e. } s \in S .
$$

Proof. Let $\Gamma$ be a dense countable subgroup of $G$. For $n \geq 1$, let $\lambda_{n}: S \rightarrow E_{1}^{*}$ be defined by

$$
\lambda_{n}(s)=R_{\gamma_{n}} f(s, \cdot) \in E_{1}^{*}=L^{\infty}(G)_{1} .
$$

By Lemma 3.10 and [Z2], Lemma 2.2, $\left\{A_{s}\right\}_{s \in S}$ is a Borel field of compact convex subsets of $L^{\infty}(G)_{1}$ and (ii) is proved.

For $f \in L^{\infty}(G), s \in S, g$ and $h \in G$, we have

$$
c^{*}(s, g) R_{h} f(s g, \cdot)=R_{h} f(s g, \cdot g)=f(s g h, \cdot g h)=R_{g h} f(s, \cdot) .
$$

Therefore, $\left\{A_{s}\right\}_{s \in S}$ is $c$-invariant.

Lemma 3.14. Let $\phi \in C_{c}(G)$ and $f \in R_{\phi}\left(L^{\infty}(S \times G)\right)$. If $(S, \mu)$ is an amenable $G$-space, then there exists $\Phi \in \overline{\mathscr{R}}$ such that $\Phi f$ is $R_{G}$-invariant.

Proof. We may assume that $\|f\|_{L^{\infty}(S \times G)} \leq 1$ and, by Lemma 3.9, that $f$ is uniformly continuous on $R$-orbits and

$$
|f(s, h)| \leq 1, \quad(s, h) \in S \times G .
$$

Let $c: S \times G \rightarrow \operatorname{Iso}\left(L^{1}(G, \rho)\right)$ and $\left\{A_{s}\right\}_{s \in S}$ be as in Lemma 3.12. As $(S, \mu)$ is an amenable $G$-space, there exists a $c$-invariant Borel section $\sigma$ in $\left\{A_{s}\right\}_{s \in S}$, i.e. a Borel function $\sigma: S \rightarrow L^{\infty}(G)_{1}$ such that $\sigma(s) \in A_{s}$ a.e. and for each $g \in G, c^{*}(s, g) \sigma(s g)=\sigma(s)$ a.e.

By Lemma 3.13, there exists $f^{\prime} \in \overline{\mathscr{R} f}$ such that

$$
\sigma(s)=f^{\prime}(s, \cdot) \text { for a.e. } s \in S \text {. }
$$

By $c$-invariance of $\sigma$, we have for each $g \in G$,

$$
R_{g} f^{\prime}(s, \cdot)=f^{\prime}(s, \cdot) \text { for a.e. } s \in S .
$$

Therefore, $f^{\prime}$ is $R_{G}$-invariant and by Remark 3.12, $f^{\prime} \in \overline{\mathscr{R}} f$.

Lemma 3.15. If $(S, \mu)$ is an amenable G-space, then there exists $\Phi \in \overline{\mathscr{R}}$ such that $\Phi f$ is $R_{G}$-invariant for all $f \in L^{\infty}(S \times G)$.

Proof. For $f \in L^{\infty}(S \times G)$, set $K_{f}=\left\{\Phi \in \overline{\mathscr{R}} ; \Phi f\right.$ is $R_{G}$-invariant $\}$. Denote by $\mathscr{K}$ the system of the closed subsets $K_{f}, f \in L^{\infty}(S \times G)$. 
As $\overline{\mathscr{R}}$ is $\sigma\left(B\left(L^{\infty}(S \times G)\right), B\left(L^{\infty}(S \times G)\right)_{*}\right)$-compact, showing that $\mathscr{K}$ has the finite intersection property will prove 3.14 .

The proof will be by induction. Let $f_{1}, \cdots, f_{k} \in L^{\infty}(S \times G)$ and $\Psi \in \overline{\mathscr{R}}$ be such that $\Psi f_{1}, \cdots, \Psi f_{k-1}$ are all $R_{G}$-invariant.

For $1 \leq i \leq k-1$, by definition of $\mathscr{R}, \Omega \Psi f_{i}=\Psi f_{i}$ for all $\Omega \in \mathscr{R}$, and therefore for all $\Omega \in \overline{\mathscr{R}}$. In particular, $\Omega \Psi f_{i}$ is $R_{G}$-invariant.

Fix a nonnegative function $\phi$ in $C_{c}(G)$ such that $\|\phi\|_{L^{1}(G, \rho)}=1$. Let $\left(\mu_{i}\right)$ be a sequence of finitely supported probability measures on $G$ such that $\mu_{i} \rightarrow \phi$. By Lemma 3.2, $R_{\mu_{i}} \rightarrow R_{\phi}$. For each $i \geq 1, R_{\mu_{i}} \in \mathscr{R}$. Therefore, $R_{\phi} \in \overline{\mathscr{R}}$.

By Lemma 3.13, there exists $\Psi^{\prime} \in \overline{\mathscr{R}}$ such that $\Psi^{\prime}\left(R_{\phi} \Psi f_{k}\right)$ is $R_{G}$-invariant. Set $\Phi=\Psi^{\prime} R_{\phi} \Psi$. Then $\Phi f_{i}$ is $R_{G}$-invariant for $1 \leq i \leq k$ and so $\mathscr{K}$ has the finite intersection property.

Proof of Theorem 3.6. Set $\mathscr{S}_{1}=\left\{\Phi \in B\left(L^{\infty}(S \times G)\right) ; \Phi(1)=1, \Phi(f) \geq\right.$ 0 , for all $\left.f \in L^{\infty}(S \times G)\right\}$.

As $|f| \leq\|f\|_{1}$, for $f \in L^{\infty}(S \times G)$, and $\Phi(1)=1$, we have

$$
\|\Phi\| \leq 1, \quad \text { for all } \Phi \in \mathscr{S}_{1} \text {. }
$$

Therefore $\mathscr{S}_{1}$ is $\sigma\left(B\left(L^{\infty}(S \times G)\right), B\left(L^{\infty}(S \times G)\right)_{*}\right)$-compact.

By Remark 3.1, the set

$$
\mathscr{S}=\left\{\Phi \in \mathscr{S}_{1} ; \Phi \text { is } L^{\infty}(S \times G)^{R} \text {-linear and } V \text {-equivariant }\right\}
$$

is $\sigma\left(B\left(L^{\infty}(S \times G)\right), B\left(L^{\infty}(S \times G)\right)_{*}\right)$-closed and therefore compact with respect to the $\sigma\left(B\left(L^{\infty}(S \times G)\right), B\left(L^{\infty}(S \times G)\right)_{*}\right)$-topology.

By definition, $\mathscr{R} \subset \mathscr{S}$. By Lemma 3.15, there exists a $R$-invariant $\Phi$ in $\overline{\mathscr{R}}$ and therefore in $\mathscr{S}$. This finishes the proof of Theorem 3.6.

\section{AMENABILITY OF STABILIZERS}

Let $(G, \rho)$ be a locally compact, second countable topological group, with a right invariant Haar measure $\rho$ as above.

In the first two lemmas, we give a necessary and sufficient condition for a closed subgroup of $G$ to be amenable.

Let $G$ act on the left and right of $L^{\infty}(G)$ by, for $f \in L^{\infty}(G)$, $g \cdot f(h)=V_{g} f(h)=f\left(g^{-1} h\right), \quad f \cdot g(h)=R_{g^{-1}} f(h)=f\left(h g^{-1}\right), \quad h \in G$.

This last action is the dual of the action of $G$ on $L^{1}(G, \rho)$ given by

$$
\chi \cdot g(h)=R_{g^{-1}} \chi(h)=\chi\left(h g^{-1}\right) \text { for } \chi \in L^{1}(G, \rho) .
$$

There is a corresponding left action of $G$ on $L^{1}(G, \rho)$ given by

$$
g \cdot \chi(h)=V_{g} \chi(h)=\chi\left(g^{-1} h\right) \Delta\left(g^{-1}\right), \quad g \in G, \chi \in L^{1}(G, \rho)
$$

where $\Delta$ is the modular function on $G$.

We will denote by $\langle\cdot, \cdot\rangle$ the pairing between $L^{1}(G, \rho)$ and $L^{\infty}(G, \rho)$ given by the integral.

Let $U C B(G)$ denote the left uniformly continuous bounded functions on $G$, i.e. the functions $f \in L^{\infty}(G)$ such that for all $\varepsilon>0$, there exists a neighbourhood $U$ of the identity in $G$ with the following property:

$$
\text { for all } g \in U, \quad\|(g \cdot f)-f\|_{L^{\infty}(G)}<\varepsilon,
$$

where $(g \cdot f)(h)=f\left(g^{-1} h\right), h \in G$. 
Set

$$
P(G)=\left\{\chi \in L^{1}(G, \rho) ; \chi \geq 0,\|\chi\|_{L^{1}(G, \rho)}=1\right\} .
$$

Definition 4.1. Let $G_{0}$ be a closed subgroup of $G$ and let $E \subseteq L^{\infty}(G)$. We will say that $E$ is a $G_{0}$-domain if $E$ is a left $G_{0}$-invariant separable norm-closed subspace of $U C B(G)$ which contains the constant functions. We denote the set of $G_{0}$-domains by $\mathscr{D}\left(G_{0}\right)$.

Lemma 4.2. Let $G_{0}$ be a closed subgroup of $G$ and $\Lambda$ a countable norm-dense subset of $G_{0}$. Let $E \in \mathscr{D}\left(G_{0}\right)$ and $E_{0}$ be a countable dense subset of $E$.

Then the two following conditions are equivalent:

(i) There is a left $G_{0}$-invariant mean on $E$.

(ii) For any finite subset $\Lambda_{0} \subseteq \Lambda$, for any finite subset $F \subseteq E_{0}$, for all $\varepsilon>0$, there exists $\psi \in \mathscr{P}(G)$ such that:

$$
\text { for all } \beta \in F \text {, and } \lambda \in \Lambda_{0}, \quad|\langle\lambda \cdot \psi-\psi, \beta\rangle| \leq \varepsilon\|\beta\|_{L^{\infty}(G)} \text {. }
$$

Proof. By continuity, the existence of a left $G_{0}$-invariant mean on $E$ is equivalent to the existence of a mean $m$ on $E$ such that: for all $\lambda \in \Lambda$, for all $e \in E_{0}$, we have $m(\lambda \cdot e)=m(e)$. The result follows from [Z4], Proposition 7.2.3(b), and the definition of the weak * topology.

Lemma 4.3. Let $G_{0}$ be a closed subgroup of $G$. Then $G_{0}$ is amenable if and only if, for every $E \in \mathscr{D}\left(G_{0}\right)$, there is a left $G_{0}$-invariant mean on $E$.

Proof. Only if: Let $U C B\left(G_{0}\right)$ denote the left uniformly continuous bounded functions in $L^{\infty}\left(G_{0}\right)$. Let $m_{0}$ be a left $G_{0}$-invariant mean on $U C B\left(G_{0}\right)$. Let $m$ denote the mean on $U C B(G)$ defined by $m(f)=m_{0}\left(f \mid G_{0}\right)$. Then, for all $E \in \mathscr{D}\left(G_{0}\right), m \mid E$ will be a left $G_{0}$-invariant mean on $E$.

If: For all $E \in \mathscr{D}\left(G_{0}\right)$, let $K_{E}$ denote the set of means on $L^{\infty}(G)$ such that $m \mid E$ is left $G_{0}$-invariant. Then $\mathscr{K}=\left\{K_{E} ; E \in \mathscr{D}\left(G_{0}\right)\right\}$ satisfies the finite intersection property; choose $m \in \bigcap \mathscr{K}$. Then $m \mid U C B(G)$ is left $G_{0^{-}}$ invariant.

Next, we mimic the argument of [Z4], Lemma 7.2 .8 , to produce a left $G_{0^{-}}$ invariant mean on $L^{\infty}(G)$.

Fix a right invariant Haar measure $\rho_{0}$ on $G_{0}$. Set

$$
P\left(G_{0}\right)=\left\{\phi \in L^{1}\left(G_{0}, \rho_{0}\right) ; \phi \geq 0,\|\phi\|_{L^{1}\left(G_{0}, \rho_{0}\right)}=1\right\} .
$$

Using [Z4], Proposition 7.2.6(c), choose an approximation $\left\{e_{n}\right\}_{n \geq 1} \subset \mathscr{P}(G)$ to the identity. If $\psi \in \mathscr{P}(G), \phi \in \mathscr{P}\left(G_{0}\right)$ and $f \in L^{\infty}(G)$, then we define $\psi \cdot \phi \in \mathscr{P}(G)$ and $\phi \cdot f \in L^{\infty}(G)$ by

$$
\begin{aligned}
& (\psi \cdot \phi)(g)=\int\left(\psi \cdot g_{0}\right)(g) \phi\left(g_{0}\right) d \rho_{0}\left(g_{0}\right), \\
& (\phi \cdot f)(g)=\int \phi\left(g_{0}\right)\left(g_{0} \cdot f\right)(g) d \rho_{0}\left(g_{0}\right) .
\end{aligned}
$$

If $\psi \in P(G)$ and $f \in L^{\infty}(G)$, then we define $\psi * f \in U C B(G)$ by

$$
(\psi * f)(h)=\int \psi(g) f\left(g^{-1} f\right)(h) d \rho(g) .
$$

Choose $\psi_{0} \in P(G)$ and consider the mean $\tilde{m}$ on $L^{\infty}(G)$ defined by $\tilde{m}(f)=$ $m\left(\psi_{0} * f\right)$. 
Fix any $f \in L^{\infty}(G)$ and $\phi \in \mathscr{P}\left(G_{0}\right)$. For all $n \in \mathbb{N}$, set $f_{n}=e_{n} * f$. By parts (a) and (c) of [Z4], Proposition 7.2.6, we have that $\psi_{0} * f_{n} \rightarrow \psi_{0} * f$ and $\left(\psi_{0} \cdot \phi\right) * f_{n} \rightarrow\left(\psi_{0} \cdot \phi\right) * f$ in $L^{\infty}(G)$ as $n \rightarrow \infty$. Now $\psi_{0} *(\phi \cdot f)=\left(\psi_{0} \cdot \phi\right) * f$ and, for all $n \in \mathbb{N}$, we have $\psi_{0} *\left(\phi \cdot f_{n}\right)=\left(\psi_{0} \cdot \phi\right) * f_{n}$. So $\tilde{m}\left(\phi \cdot f_{n}\right) \rightarrow \tilde{m}(\phi \cdot f)$. Further, $\tilde{m}\left(f_{n}\right) \rightarrow \tilde{m}(f)$. But the argument of [Z4], Proposition 7.2.7, shows, for all $n \in \mathbb{N}$, that $\tilde{m}\left(\phi \cdot f_{n}\right)=\tilde{m}\left(f_{n}\right)$. We conclude that $\tilde{m}(\phi \cdot f)=\tilde{m}(f)$.

Fix any $f \in L^{\infty}(G)$ and $g_{0} \in G_{0}$. Choose any $\phi \in P\left(G_{0}\right)$. By the argument of the last paragraph, $\tilde{m}\left(\phi \cdot\left(g_{0} \cdot f\right)\right)=\tilde{m}\left(g_{0} \cdot f\right)$ and $\tilde{m}\left(\left(\phi \cdot g_{0}\right) \cdot f\right)=\tilde{m}(f)$. But $\phi \cdot\left(g_{0} \cdot f\right)=\left(\phi \cdot g_{0}\right) \cdot f$, so we conclude that $\tilde{m}\left(g_{0} \cdot f\right)=\tilde{m}(f)$. That is, $\tilde{m}$ is a left $G_{0}$-invariant mean on $L^{\infty}(G)$.

Let $s: G_{0} \backslash G \rightarrow G$ be a Borel section of the natural map $\pi: G \rightarrow G_{0} \backslash G$. Define $p: G \rightarrow G_{0}$ by $p(g)=g[s(\pi(g))]^{-1}$. For all $g \in G$, for all $g_{0} \in G_{0}$, we have $p\left(g_{0} g\right)=g_{0} p(g)$. Define a mean $m_{0}$ on $L^{\infty}\left(G_{0}\right)$ by $m_{0}(f)=\tilde{m}(f \circ p)$; then $m_{0}$ is left $G_{0}$-invariant.

Let $(S, \mu)$ be a standard Borel $G$-space with a quasi-invariant probability measure $\mu$. We denote by $\mathscr{R}_{G}$ the equivalence relation induced by the action of $G$ on $(S, \mu)$.

Recall that a complete lacunary section $T \subset S$ is a Borel set such that $T G$ is conull and there is a neighbourhood $U$ of the identity of $G$ such that

$$
s U \cap T=\{s\} \text { for all } s \in S .
$$

Clearly every lacunary section is a countable section (i.e. $s G \cap T$ is countable for all $s \in S$ ).

Proposition 4.4. There exist

(1) a Borel set $T \subseteq S$,

(2) a probability measure $\nu$ on $T$,

(3) a Borel map $\phi: S \rightarrow G$, and

(4) a Borel map $\sigma: \mathscr{R}_{G} \mid T \rightarrow G$

such that:

(A) for all $s \in S,(s G) \cap T$ is countable and nonempty;

(B) for all Borel subsets $T_{0} \subseteq T$, we have: $T_{0}$ is $\nu$-null if and only if $T G_{0}$ is $\mu$-null;

(C) for all $s \in S, s \phi(s) \in T$;

(D) for all $t \in T, \phi(t)=1_{G}$; and

(E) for all $\left(t, t^{\prime}\right) \in \mathscr{R}_{G} \mid T, t \sigma\left(t, t^{\prime}\right)=t^{\prime}$.

Proof. By [Ke], Corollary 1.2, there exists a complete lacunary section $T \subseteq S$ for the $G$-action.

Set $A=\{(s, g) \in S \times G ; s g \in T\}$ and $A_{s}=\{g \in G ;(s, g) \in A\}$. Let us show that for each $s \in S, A_{s}$ is a closed subset of $G$.

Fix $s \in S$ and let $\left(g_{n}\right)_{n \geq 1}$ be a converging sequence in $G$ whose limit is $g \in G$ and such that $s g_{n} \in T$, for all $n \geq 1$. Choose a neighbourhood $U$ of the identity in $G$ such that

$$
t U \cap T=\{t\} \text { for all } t \in T .
$$

Choose a neighbourhood $V$ of the identity in $G$ such that $V^{-1} V \subset U$. We may assume that for all $n, g_{n} \in g V$. Then for each $n$ and $m$, we have

$$
\left(s g_{n}\right)\left(g_{n}^{-1} g_{m}\right)=s g_{m} \in T \text { and } g_{n}^{-1} g_{m} \in(g V)^{-1}(g V)=V^{-1} V \subset U \text {, }
$$


so $s g_{m}=\left(s g_{n}\right)\left(g_{n}^{-1} g_{m}\right) \in\left(s g_{n}\right) U \cap T=\left\{s g_{n}\right\}$. Thus, for all $n$ and $m$, we have $s\left(g_{m} g_{n}^{-1}\right)=s$. By [Z4], Corollary 2.1.20, $G_{s}$ is closed in $G$, and therefore $g g_{n}^{-1}$ belongs to $G_{s}$. Hence $s g=s g_{n} \in T$ which proves that $A_{s}$ is closed.

Since $G$ is second countable and locally compact, $A_{s}$ is $\sigma$-compact for every $s \in S$. By a standard theorem in Borel selection theory (see [Ka], Proposition 3.1), we may therefore choose a Borel map $\phi: S \rightarrow G$ such that

$$
(\phi(s), s) \in A \text { for all } s \in S \text {. }
$$

Then for all $s \in S$, we have $\phi(s) \in A_{s}$, i.e. $s \phi(s) \in T$.

Let $\nu_{0}$ denote the image of the measure $\mu$ on $S$ under the map from $S$ to $T$ given by $s \mapsto s \phi(s)$.

By [FM], Theorem 1, choose a countable group $\Lambda$ and a Borel action of $\Lambda$ on $T$ such that $R \mid T=\{(t, t \lambda) \mid t \in T, \lambda \in \Lambda\}$. Let $\left\{\lambda_{n}\right\}_{n \geq 1}$ be a listing of the elements of $\Lambda$. Let $\nu_{i}$ be the image of $\nu_{0}$ under the map from $T$ to $T$ given by $t \mapsto t \lambda_{i}$. Set $\nu=\sum_{i=1}^{\infty}\left(2^{-i}\right) \nu_{i}$.

Lemma 4.5. There is a sequence $\left(\phi_{n}\right)_{n \geq 1}$ of Borel functions from $S$ to $G$ such that, for all $s \in S$, the set $\left(\phi_{n}(s)\right)_{n \geq 1}$ is a dense subset of $G_{s}$.

Proof. Choose a sequence $\left(U_{n}\right)_{n>1}$ of precompact open sets in $G$ such that if $g \in G$ and if $V$ is any neighbourhood in $G$ of $g$, then, for some $i \in \mathbb{N}$, we have $g \in U_{i} \subseteq \overline{U_{i}} \subseteq V$.

Set $X=\{(s, g) \in S \times G ; s g=s\}$. Let $p: S \times G \rightarrow S$ denote the first coordinate projection. Let $q: S \times G \rightarrow G$ denote the second coordinate projection. For all $i \in \mathbb{N}$, set $X_{i}=X \cap\left(S \times \overline{U_{i}}\right)$ and set $S_{i}=p\left(X_{i}\right)$.

Fix $i \in \mathbb{N}$. By a theorem in Borel selection theory (see, for example [Ka], Proposition 3.1), it follows that $S_{i}$ is a Borel subset of $S$ and that there is a Borel map $x_{i}: S_{i} \rightarrow X_{i}$ such that: for all $s \in S_{i}$, we have $p\left(x_{i}(s)\right)=s$. Let $\phi_{i}: S \rightarrow G$ be defined by $\phi_{i}(s)=q(x(s))$, for all $s \in S_{i}$, and $\phi_{i}(s)=1_{G}$, for all $s \in S \backslash S_{i}$.

Let $U C B_{1}^{+}(G)$ denote the set of nonnegative functions $f \in U C B(G)$ such that $\|f\|_{L^{\infty}(G)}=1$. Give $U C B_{1}^{+}(G) \subseteq L^{1}(G, \rho)^{*}$ the weak *topology.

Let $Y=U C B_{1}^{+}(G)^{\mathbb{N}}$ denote the countable Cartesian product of copies of $U C B_{1}^{+}(G)$ and give $Y$ the product topology. Let 1 denote the constant function on $G$ which is identically equal to 1 . Set $Y_{1}=\left\{\left(\beta_{1}, \beta_{2}, \ldots\right) \in Y ; \beta_{1}=\right.$ $1\}$.

For all $\beta=\left(\beta_{1}, \beta_{2}, \ldots\right) \in Y_{1}$, let $E(\beta)$ denote the norm closure in $L^{\infty}(G)$ of the span of $\left\{\beta_{n}\right\}_{n \geq 1}$.

Lemma 4.6. Let $\mathscr{E}^{\prime} \subseteq S \times Y_{1}$ denote the set of all $(s, \beta)$ such that $E(\beta)$ is left $G_{s}$-invariant. Let $\mathscr{E}$ denote the set of all $(s, \beta) \in \mathscr{E}^{\prime}$ such that $E(\beta)$ admits a left $G_{s}$-invariant mean. Then $\mathscr{E}^{\prime}$ and $\mathscr{E}$ are Borel subsets of $S \times Y_{1}$.

Proof. Let $\left(\psi_{n}\right)_{n \geq 1}$ be a norm dense sequence in $P(G)$ and $\left(\chi_{n}\right)_{n \geq 1}$ be one in the unit ball of $\left.L^{1}(G, \rho)\right)$.

Set $\bigoplus_{n \geq 1} \mathbb{Q}=\left\{\tau=\left(\tau_{n}\right)_{n \geq 1} ; \tau_{n}=0\right.$ for all sufficiently large $\left.n\right\}$, the direct sum of copies of the rational numbers. Let $\left(\tau^{n}\right)_{n \geq 1}$ be a listing of all the elements of $\bigoplus_{n>1} \mathbb{Q}$.

For all $\tau \in \bigoplus_{n \geq 1} \mathbb{Q}$, for all $\beta=\left(\beta_{1}, \beta_{2}, \ldots\right) \in Y_{1}$, set $\tau \cdot \beta=\sum_{i=1}^{\infty} \tau_{i} \beta_{i}$. Then, for all $\beta \in \bar{Y}_{1}$, the set $\left\{\tau^{k} \cdot \beta ; k \in \mathbb{N}\right\}$ is norm dense in $E(\beta)$. 
By Lemma 4.5, choose a sequence $\left(\phi_{n}\right)_{n \geq 1}$ of Borel functions from $S$ to $G$ such that, for all $s \in S$, the set $\left\{\phi_{i}(s) ; i \in \mathbb{N}\right\}$ is a dense subset of $G_{s}$.

Fix $(s, \beta) \in S \times Y_{1}$. Then

$(s, \beta) \in \mathscr{E}^{\prime}$ if and only if $\forall i, j \in \mathbb{N}, \phi_{i}(s) \cdot \beta_{j} \in E(\beta)$

if and only if

$\forall i, j, n \in \mathbb{N}, \exists k \in \mathbb{N}$ such that $\left\|\phi_{i}(s) \cdot \beta_{j}-\tau^{k} \cdot \beta\right\|_{L^{\infty}(G)}<\frac{1}{n}$,

if and only if

$\forall i, j, n \in \mathbb{N}, \exists k \in \mathbb{N}$ such that $\forall l \in \mathbb{N},\left|\left\langle\phi_{i}(s) \cdot \beta_{j}-\tau^{k} \cdot \beta, \chi_{l}\right\rangle\right|<\frac{1}{n}$.

This shows that $\mathscr{E}^{\prime}$ is Borel in $S \times Y_{1}$.

Fix $(s, \beta) \in \mathscr{E}^{\prime}$. By Lemma 4.2 and Lemma 4.3,

$(s, \beta) \in \mathscr{E}$ if and only if

$\forall i_{0}, k_{0}, n \in \mathbb{N}, \exists m \in \mathbb{N}$ such that $\forall i=1, \ldots, i_{0}, \forall k=1, \ldots, k_{0}$,

$$
\left|\left\langle\phi_{i}(s) \cdot \psi_{m}-\psi_{m}, \tau^{k} \beta\right\rangle\right| \leq \frac{1}{n}\left\|\tau^{k} \cdot \beta\right\|_{L^{\infty}(G)}
$$

if and only if

$\forall i_{0}, k_{0}, n \in \mathbb{N}, \exists m \in \mathbb{N}$ such that $\forall i=1, \ldots, i_{0}, \forall k=1, \ldots, k_{0}, \exists l \in \mathbb{N}$ with

$$
\left|\left\langle\phi_{i}(s) \cdot \psi_{m}-\psi_{m}, \tau^{k} \cdot \beta\right\rangle\right| \leq \frac{1}{n}\left|\left\langle\tau^{k} \cdot \beta, \chi_{l}\right\rangle\right| .
$$

This shows that $\mathscr{E}$ is Borel in $S \times Y_{1}$.

Lemma 4.7. Assume that the G-action on $S$ is amenable. Suppose that the map from $S$ to $Y_{1}$ given by $s \mapsto \beta^{s}$ is measurable. Suppose, for all $g \in G$, for a.e. $s \in S$, that $E\left(\beta^{s g}\right)=g \cdot E\left(\beta^{s}\right)$. Then, for a.e. $s \in S$, there is a $G_{s}$-invariant mean on $E\left(\beta^{s}\right)$.

Proof. Let $P: L^{\infty}(S \times G) \rightarrow L^{\infty}(S)$ be as in Theorem 3.4.

For all $i \in \mathbb{N}$, for all $(s, g) \in S \times G$, let $f_{i} \in L^{\infty}(S \times G)$ be defined by $f_{i}(s, g)=\beta_{i}^{s}(g)$; then $\left\|f_{i}\right\|_{L^{\infty}(S \times G)} \leq 1$, by definition of $Y_{1}$.

For all $i \in \mathbb{N}$, let $c_{i}$ be a bounded Borel function on $S$ such that $c_{i}=P\left(f_{i}\right)$ a.e. on $S$ and such that $0 \leq c_{i} \leq 1$ on $S$.

By disintegration techniques (cf. [F], Chapter 5, $\S 4$, pp. 107-110]), for a.e. $s \in$ $S$, there is a unique mean $m^{s}$ on $E\left(\beta^{s}\right)$ such that: for all $i \in \mathbb{N}$, we have $m^{s}\left(\beta_{i}^{s}\right)=c_{i}(s)$. Moreover, for all $g \in G$, for a.e. $s \in S$, for all $f \in E\left(\beta^{s}\right)$, we have $m^{s g}(g \cdot f)=m^{s}(f)$.

If $E$ is any separable norm-closed subspace of $L^{\infty}(G)$ containing the constant functions, if $m$ is a mean on $E$, and if $g \in G$, then we let $m . g$ denote the mean on $g \cdot E$ defined by $(m . g)(f)=m\left(g^{-1} \cdot f\right)$. With this notation, for all $g \in G$, for a.e. $s \in S$, we have $m^{s} . g=m^{s g}$.

Thus, for a.e. $s \in S$, for a.e. $g \in G$, we have $m^{s} . g=m^{s g}$. So, for a.e. $s \in S$, for all $g^{\prime} \in G$, for a.e. $g \in G$, we have

$$
m^{s} . g=m^{s g}, \quad m^{s} \cdot\left(g g^{\prime}\right)=m^{s g g^{\prime}} .
$$

Therefore, for a.e. $s \in S$, for all $g^{\prime} \in G_{s}$, for a.e. $g \in G$, we have

$$
m^{s g} \cdot\left(g^{-1} g^{\prime} g\right)=\left(m^{s} \cdot g\right) \cdot\left(g^{-1} g^{\prime} g\right)=m^{s} \cdot\left(g^{\prime} g\right)=m^{s\left(g^{\prime} g\right)}=m^{s g} \text {. }
$$


Then, for a.e. $s \in S$, for a.e. $g \in G$, for a.e. $g^{\prime} \in G_{s g}$, we have $m^{s g} \cdot g^{\prime}=m^{s g}$. Then there exists $g_{0} \in G$ such that: for a.e. $s \in S$, for a.e. $g^{\prime} \in G_{s g_{0}}$ we have $m^{s g_{0}} \cdot g=m^{s g_{0}}$. For a.e. $s \in S$, this implies: for a.e. $g \in G_{s}$, we have $m^{s} . g=m^{s}$, i.e., $m^{s}$ is invariant under a conull set in $G_{s}$.

So, for a.e. $s \in S$, the set of elements of $G_{s}$ fixing $m^{s}$ is a conull subgroup of $G_{s}$; by [Z4], Proposition B.1, $m^{s}$ is $G_{s}$-invariant.

Lemma 4.8. The set of all $s \in S$ such that $G_{s}$ is amenable is a measurable subset of $S$.

Proof. Let $\mathscr{E}^{\prime}, \mathscr{E} \subseteq S \times Y_{1}$ be as in Lemma 4.6 ; then $\mathscr{E}^{\prime}$ and $\mathscr{E}$ are Borel subsets of $S \times Y_{1}$. Let $\pi_{1}: S \times Y_{1} \rightarrow S$ denote the projection onto the first coordinate.

By Lemma 4.3, the set of $s \in S$ such that $G_{s}$ is nonamenable is equal to $\pi_{1}\left(\mathscr{E}^{\prime} \backslash \mathscr{E}\right)$. This set is analytic, hence measurable ([A], Theorem 3.2.4). Consequently, its complement, which is the set in question, is also measurable.

A. Kechris informs us that the set where stabilizers are amenable is actually Borel.

Lemma 4.9. Let $S_{1}$ be a measurable G-invariant subset of $S$. Then there exists a Borel G-invariant subset $S_{0}$ of $S$ such that $S_{0} \subseteq S_{1}$ and such that $\mu\left(S_{1} \backslash S_{0}\right)=$ 0 .

Proof. Let $S_{1}^{\prime}$ be any Borel subset of $S$ such that $S_{1}^{\prime} \subseteq S_{1}$ and such that $\mu\left(S_{1} \backslash S_{1}^{\prime}\right)=0$. Then $S_{1}^{\prime}$ is essentially $G$-invariant, in the sense that, for all $g \in G$, the symmetric difference $\left(S_{1}^{\prime} g\right) \Delta S_{1}^{\prime}$ is $\mu$-null.

In [M], $\S 6$, Theorem 3 , it is proved that an essentially $G$-invariant Borel set $E$ differs by a null set from a Borel set $D$ which is invariant under $G$. An examination of the proof shows that $D \subseteq E$.

Applying this with $E=S_{1}^{\prime}$, and taking $S_{0}$ to be the resulting set $D$, we obtain the desired conclusion.

Theorem 4.10. Assume that the G-action on $S$ is amenable. Then, for a.e. $s \in$ $S$, the group $G_{s}$ is amenable.

Proof. Let $\mathscr{E}^{\prime}, \mathscr{E} \subseteq S \times Y_{1}$ be as in Lemma 4.6. Let $\pi_{1}: S \times Y_{1} \rightarrow S$ and $\pi_{2}: S \times Y_{1} \rightarrow Y_{1}$ be the projections onto the first and second coordinates. By Lemma $4.3, \pi_{1}\left(\mathscr{E}^{\prime} \backslash \mathscr{E}\right)$ is the set of $s \in S$ such that $G_{s}$ is nonamenable. In particular, $\pi_{1}\left(\mathscr{E}^{\prime} \backslash \mathscr{E}\right)$ is $G$-invariant. Assume that $\pi_{1}\left(\mathscr{E}^{\prime} \backslash \mathscr{E}\right)$ has positive measure; we wish to obtain a contradiction.

Using Lemma 4.9, choose a $G$-invariant Borel set $S_{0}$ of positive measure in $S$ such that $S_{0} \subseteq \pi_{1}\left(\mathscr{E}^{\prime} \backslash \mathscr{E}\right)$. Replacing $S$ by $S_{0}$, we may assume, for all $s \in S$, that $G_{s}$ is nonamenable, i.e. we may assume that $\pi_{1}\left(\mathscr{E}^{\prime} \backslash \mathscr{E}\right)=S$.

By [A], Theorem 3.4.3, choose a measurable map $x: S \rightarrow \mathscr{E}^{\prime} \backslash \mathscr{E}$ such that

$$
\pi_{1}(x(s))=s, \quad \text { for all } s \in S .
$$

For $s \in S$ and $j \in \mathbb{N}$, let $x_{j}(s) \in U C B_{1}^{+}(G)$ denote the $j$ th coordinate of $\pi_{2}(s(x)) \in Y_{1}=U C B_{1}^{+}(G)^{\mathbb{N}}$.

Let $T, \nu, \phi, \sigma$ be as in Proposition 4.5. Using [FM], Theorem 1, choose a countable group $\Lambda$ and a Borel action of $\Lambda$ on $T$ such that, for all $t \in T$, 
we have $(t G) \cap T=t \Lambda$. Let $\left\{\left(\lambda_{n}, j_{n}\right)\right\}_{n \geq 1}$ be a listing of all the elements of $\Lambda \times \mathbb{N}$.

Let 1 denote the constant function on $G$ which is identically equal to 1 .

For all $t \in T$, for all $i \in \mathbb{N}$, set $\tilde{\beta}_{i}^{t}=s_{j_{i}} \sigma\left(t \lambda_{i}, t\right)\left(t \lambda_{i}\right) \in U C B_{1}^{+}(G)$. For all $s \in S$, for all $i \in \mathbb{N}$, set $\beta_{i}^{s}=\tilde{\beta}_{i}^{s \phi(s)}[\phi(s)]^{-1}$. For each $s \in S$, $\beta^{s}=\left(1, \beta_{1}^{s}, \beta_{2}^{s}, \ldots\right) \in Y_{1}$ and $s \mapsto \beta^{s}$ is measurable. By Lemma 4.7, for a.e. $s \in S$, there is a $G_{s}$-invariant mean on $E\left(\beta^{s}\right)$, and hence on $E\left(\pi_{2}(x(s))\right)$, since $E\left(\pi_{2}(x(s))\right) \subseteq E\left(\beta^{s}\right)$. But then, for a.e. $s \in S$, we have $x(s) \in \mathscr{E}$, contradicting the definition of $x$.

\section{Proof of the implication From (v) TO (i)}

Let $(S, \mu)$ be a $G$-space and $\mathscr{R}_{G}$ be the equivalence relation induced by the action of $G$. For all $s \in S$, the closed subgroup $G_{s}=\{g \in G ; s g=s\}$ denotes the stabilizer in $G$ of $s$.

Theorem 5.1. If $\mathscr{R}_{G}$ is an amenable equivalence relation on $(S, \mu)$ and if the stabilizer $G_{s}$ is amenable $\mu$-a.e., then $(S, \mu)$ is an amenable $G$-space.

Proof. Let $E$ be a separable Banach space and $E_{1}^{*}$ be the unit ball in the dual, endowed with the $\sigma\left(E^{*}, E\right)$-topology. Let $H\left(E_{1}^{*}\right)$ denote the group of homeomorphisms of $E_{1}^{*}$ with the topology of uniform convergence. Let $\alpha$ : $S \times G \rightarrow \operatorname{Iso}(E)$ be a Borel cocycle and let $\alpha^{*}: S \times G \rightarrow H\left(E_{1}^{*}\right)$ denote the induced adjoint (Borel) cocycle defined by

$$
\alpha^{*}(s, g)=\left(\alpha(s, g)^{-1}\right)^{*} .
$$

Let $\left\{A_{s}\right\}_{s \in S}$ be an $\alpha$-invariant Borel field of nonempty compact convex subsets of $E_{1}^{*}$, i.e. for each $g \in G, \alpha^{*}(s, g) A_{s g}=A_{s}$, for almost all $s \in S$.

We wish to show that there is an $\alpha$-invariant section $\psi$ in $\left\{A_{s}\right\}_{s \in S}$ (i.e. a Borel map $\psi: S \rightarrow E_{1}^{*}$ such that $\psi(s) \in A_{s}$ a.e. and for each $g \in$ $G, \alpha^{*}(s, g) \psi(s g)=\psi(s)$ a.e. $)$.

By [Z4], Theorem B.9, we may assume that the cocycle $\alpha$ is a strict cocycle. By Lemma 4.9 , we may replace $S$ by a $G$-invariant conull Borel subset and assume: for all $s \in S$, for a.e. $g \in G$, we have $\alpha^{*}(s, g) A_{s g}=A_{s}$. Similarly, we may assume, for all $s \in S$, that $G_{s}$ is amenable.

Fix $s \in S$ and $g \in G$. Choose $g^{\prime} \in G$ such that

$$
\alpha^{*}\left(s, g g^{\prime}\right) A_{s g g^{\prime}}=A_{s} \quad \text { and } \quad \alpha^{*}\left(s g, g^{\prime}\right) A_{s g g^{\prime}}=A_{s g} .
$$

Then by the cocycle identity, we have

$$
\alpha^{*}(s, g) A_{s g}=\alpha^{*}(s, g) \alpha^{*}\left(s g, g^{\prime}\right) A_{s g g^{\prime}}=\alpha^{*}\left(s, g g^{\prime}\right) A_{s g g^{\prime}}=A_{s} .
$$

For all $s \in S$, let $G_{s}$ act on $A_{s}$ by $a \cdot g=\alpha^{*}(s, g) a$ and let $B_{s}=A_{s}^{G_{s}}$ denote the $G_{s}$ fixed points in $A_{s}$. By amenability of the stabilizers, for all $s \in S, B_{s} \neq \varnothing$.

Choose $T, \nu, \phi, \sigma$ as in Proposition 4.4. By [CFW], there is a $\nu$-conull, $\left(\mathscr{R}_{G} \mid T\right)$-invariant subset $T_{0} \subseteq T$ and a Borel $\mathbb{Z}$-action on $T_{0}$ such that

$$
\mathscr{R}_{G} \mid T_{0}=\left\{(t, t . n) \mid t \in T_{0}, n \in \mathbb{Z}\right\} .
$$

By Lemma 4.9, choose a $\mu$-conull, Borel, $G$-invariant subset $S_{0}$ of $T_{0} G$. Replacing $T$ by $S_{0} \cap T$ and $S$ by $S_{0}$, we may assume that $T_{0}=T$. 
Define $\alpha_{0}:\left(\mathscr{R}_{G} \mid T\right) \rightarrow \operatorname{Iso}(E)$ by $\alpha_{0}\left(t, t^{\prime}\right)=\alpha\left(t, \sigma\left(t, t^{\prime}\right)\right)$. Let $\alpha_{0}^{*}$ : $\left(\mathscr{R}_{G} \mid T\right) \rightarrow H\left(E_{1}^{*}\right)$ be defined by

$$
\alpha_{0}^{*}\left(t, t^{\prime}\right)=\left(\alpha_{0}\left(t, t^{\prime}\right)\right)^{*} .
$$

For all $t \in T$, for all $t^{\prime} \in(G t) \cap T$, we have $\alpha_{0}^{*}\left(t^{\prime}, t\right) B_{t}=B_{t^{\prime}}$. Moreover, for all $t \in T$, for all $t^{\prime}, t^{\prime \prime} \in(t G) \cap T$, for all $b \in B_{t}$, we have

$$
\alpha_{0}^{*}\left(t^{\prime \prime}, t^{\prime}\right) \alpha_{0}^{*}\left(t^{\prime}, t\right) b=\alpha_{0}^{*}\left(t^{\prime \prime}, t\right) b .
$$

Let $K$ denote the set of all $\psi \in L^{\infty}\left((T, \nu), E^{*}\right)$ such that, for $\nu$-a.e. $t \in$ $T$, we have $\psi(t) \in B_{t}$. Then $K$ is a weak * compact convex subset of $L^{\infty}\left((T, \nu), E^{*}\right) \cong L^{1}((T, \nu), E)^{*}$. Let $\mathbb{Z}$ act on $K$ by the rule

$$
(\psi \cdot n)(t)=\alpha_{0}^{*}(t, t n) \psi(t n) .
$$

Using [A], Theorem 3.4.3, choose $\psi_{0} \in L^{\infty}\left((T, \nu), E^{*}\right)$ such that $\psi_{0}(t) \in$ $B_{t}$, for a.e. $t \in T$. That is, $\psi_{0} \in K$. For every $N \in \mathbb{N}$, set

$$
\psi_{N}=\frac{1}{2 N+1} \sum_{n=-N}^{N} \psi_{0} \cdot n \text {. }
$$

Let $\tilde{\psi}$ be an accumulation point in $K$ of $\left\{\psi_{N} ; N \in \mathbb{N}\right\}$. For $\nu$-a.e. $t \in$ $T, \tilde{\psi}(t) \in B_{t}$. Further, for $\nu$-a.e. $t \in T$, for all $t^{\prime} \in(t G) \cap T$, we have $\alpha_{0}^{*}\left(t, t^{\prime}\right) \tilde{\psi}(t)=\tilde{\psi}\left(t^{\prime}\right)$.

Define $\psi \in L^{\infty}\left(S, E^{*}\right)$ by the rule

$$
\psi(s)=\alpha^{*}(s, \phi(s)) \tilde{\psi}(s \phi(s)) .
$$

Then, for a.e. $s \in S, \psi(s) \in B_{s} \subseteq A_{s}$. Further, for a.e. $s \in S$, for all $g \in G$, we have $\alpha^{*}(s, g) \psi(s g)=\psi(s)$, as desired.

\section{REFERENCES}

[A] W. Arveson, Invitation to C*-algebras, Springer-Verlag, Berlin, 1976.

[AS] S. Adams and G. Stuck, Splitting of non-negatively curved leaves in minimal sets of foliations, Duke Math. J. 71 (1993), 71-92.

[C] A. Connes, On hyperfinite factors of type $\mathrm{III}_{0}$ and Krieger's factors, J. Funct. Anal. 18 (1975), 318-327.

[CFW] A. Connes, J. Feldman and B. Weiss, An amenable equivalence relation is generated by a single transformation, Ergodic Theory Dynamical Systems 1 (1981), 431-450.

[CW] A. Connes and E. J. Woods, Hyperfinite von Neumann algebras and Poisson boundaries of time dependent random walks, Pacific J. Math. 137 (1989), 225-243.

[EG1] G. A. Elliott and T. Giordano, Amenable actions of discrete groups, Ergodic Theory Dynamical Systems (to appear).

[FHM] J. Feldman, P. Hahn and C. Moore, Orbit structure and countable sections for actions of continuous groups, Adv. in Math. 28 (1978), 186-230.

[FM] J. Feldman and C.C. Moore, Ergodic equivalence relations, cohomology and von Neumann algebras I, Trans. Amer. Math. Soc. 234 (1977), 289-324.

[F] H. Furstenberg, Recurrence in ergodic theory and combinatorial number theory, Princeton University Press, Princeton, NJ, 1981.

[GS] V.Ya. Golodets and S.D. Sinel'shchikov, Amenable ergodic group actions and images of cocycles, Dokl. Akad. Nauk SSSR 312 (1990), 1296-1299; transl. in Soviet Math. Dokl. 41 (1990), 523-526. 
[GS1] _ Outer conjugucy for actions of continuous amenable groups, Publ. Inst. Res. Math Sci. 23 (1987), 737-769.

[GS2] Classification and the structure of cocycles of amenable ergodic equivalence relations, preprint.

[J] W. Jaworski, Poisson and Furstenberg boundaries of random walks, Ph.D. thesis, Queen's, University at Kingston, 1991.

[Ka] R. Kallman, Certain quotient spaces are countably separated, III, J. Funct. Anal. 22 (1976), 225-241.

[Ke] A. Kechris, Countable sections for locally compact group actions, preprint.

[M] G. Mackey, Point realizations of transformations groups, Illinois J. Math 6 (1962), 327-335.

[S] C.E. Sutherland, Preliminary report on Bratteli diagrams, private communication.

[V] V.S. Varadarajan, Geometry of quantum theory, 2nd ed., Springer-Verlag, Berlin, 1985.

[Z1] R.J. Zimmer, Amenable ergodic group actions and an application to Poisson boundaries of random walks, J. Funct. Anal. 27 (1978), 350-372.

[Z2] - Hyperfinite factors and amenable ergodic actions, Invent. Math. 41 (1977), 23-31.

[Z3] _ On the von Neumann algebra of an ergodic group action, Proc. Amer. Math. Soc. 41 (1977), 23-31.

[Z4] _ Ergodic theory and semisimple groups, Birkhäuser, Boston, MA, 1984.

School of Mathematics, University of Minnesota, 127 Vincent Hall, 206 Church Street, S.E., Minneapolis, Minnesota 55455

Mathematics Institute, Universitetsparken, 2100 Copenhagen $\varnothing$, Denmark

Department of Mathematics, University of Toronto, Toronto, M5S 1A1, Canada

Department of Mathematics, University of Ottawa, 585 King Edward, Ottawa, K1N 6N5, CANADA 